\title{
Characteristics of the Lamb vector in the outer region of a turbulent boundary layer
}

\author{
B. Ganapathisubramani* \\ Department of Aeronautics, Imperial College London, \\ Prince Consort Road, London SW7 2AZ, UK
}

$\begin{array}{llll}x_{1} & \text { Streamwise direction } & x_{3} & \text { Spanwise direction } \\ x_{2} & \text { Wall-normal direction } & U_{1} & \text { Mean streamwise velocity }(\mathrm{m} / \mathrm{s}) \\ U_{3} & \text { Mean spanwise velocity }(\mathrm{m} / \mathrm{s}) & U_{2} & \text { Mean wall-normal velocity }(\mathrm{m} / \mathrm{s}) \\ u_{1} & \text { Fluctuating streamwise velocity }(\mathrm{m} / \mathrm{s}) & u_{2} & \text { Fluctuating wall-normal velocity }(\mathrm{m} / \mathrm{s}) \\ u_{3} & \text { Fluctuating spanwise velocity }(\mathrm{m} / \mathrm{s}) & u_{1} u_{2} & \text { Reynolds shear stress } \\ U_{\infty} & \text { Freestream streamwise velocity }(\mathrm{m} / \mathrm{s}) & U_{\tau} & \text { skin friction velocity }(\mathrm{m} / \mathrm{s}) \\ \delta & \text { Boundary layer thickness } & \theta & \text { Momentum thickness } \\ \omega_{1} & \text { Fluctuating streamwise vorticity }(1 / \mathrm{s}) & \omega_{2} & \text { Fluctuating wall-normal vorticity }(1 / \mathrm{s}) \\ \omega_{3} & \text { Fluctuating spanwise vorticity }(1 / \mathrm{s}) & {[\vec{u} \times \vec{\omega}]} & \text { Lamb vector }\left(\mathrm{m} / \mathrm{s}^{2}\right)\end{array}$

\section{Introduction}

A characteristic feature of wall-turbulence is the presence of 'sweeps', $u_{1}>0, u_{2}<0$, Q4 events, (where high momentum fluid is transported to the wall) and 'ejections', $u_{1}<0, u_{2}>0$, Q2 events (low momentum fluid transported away from the wall). These sweep and ejection events are found to make substantial contributions to turbulence production. ${ }^{1-4}$ In other words, these Reynolds shear stress generating events act as a source for kinetic energy to sustain turbulence. In this study, the streamwise, wall-normal and spanwise directions are along the $x_{1}, x_{2}$ and $x_{3}$ axes respectively. The mean and fluctuating velocity components along those three directions are represented by $\left(U_{1}, U_{2}, U_{3}\right)$ and $\left(u_{1}, u_{2}, u_{3}\right)$, respectively.

The flow fields that contribute to Reynolds shear stress are well documented in the literature. Theodorsen ${ }^{5}$ and Offen \& Kline $^{6}$ explained the presence of sweeps and ejections using a hairpin model. Bandyopadhyay ${ }^{7}$ and Head \& Bandyopadhyay ${ }^{8}$ found visual evidence for the existence of large structures with characteristic inclined interface. Adrian et al. ${ }^{9}$ called these large scale structures as hairpin packets that comprise a group of individual hairpin type vortices, whereas $\mathrm{Na}$ et al. ${ }^{10}$ classified large-scale low-momentum regions that produce Reynolds stress as superbursts. Ganapathisubramani et al. ${ }^{11}$ found that a signature consistent with hairpin packets, contributes close to $25 \%$ of Reynolds shear stress and yet occupy only a fraction of the area. Guala et al. ${ }^{12}$ found that very large scale motions (which can be understood as a collection of hairpin packets ${ }^{13}$ ) are very active and contain more than half the Reynolds shear stress.

While the focus on Reynolds shear stress is warranted, inspection of the mean momentum equation indicates that in fact it is the gradient of Reynolds shear stress that is of primary importance to the rate of change of momentum. The mean momentum equation along the streamwise direction (equation 1) for a zero pressure gradient turbulent boundary layer shows that the wall-normal gradient of Reynolds shear stress $(T)$ is a force that acts as a momentum source/sink.

$$
\begin{aligned}
U_{1} \frac{U_{1}}{\partial x_{1}}+U_{2} \frac{\partial U_{1}}{\partial x_{2}} & =\nu \frac{\partial^{2} U_{1}}{\partial x_{2}{ }^{2}}+\bar{T} \\
\text { where, } \quad T & =\frac{\partial\left(-u_{1} u_{2}\right)}{\partial x_{2}}
\end{aligned}
$$

${ }^{*}$ Lecturer, Department of Aeronautics, Imperial College London. Member 
where, $\nu$ is the kinematic viscosity and $\bar{T}$ is the mean value of the wall-normal gradient of Reynolds shear stress.

Examination of wall-normal variation of Reynolds shear stress in wall-bounded flows reveal a single peak position $\left(x_{2 p}\right)$ for $\overline{-u_{1} u_{2}}$, which appear to be Reynolds number dependent. At the peak, the gradient of Reynolds shear stress is zero. However, the term $T$ acts as a momentum source for $x_{2}<x_{2 p}$ (where $\left.\partial\left(-\overline{u_{1} u_{2}}\right) / \partial x_{2}>0\right)$ and as a sink for $x_{2}>x_{2 p}$ (where $\left.\partial\left(-\overline{u_{1} u_{2}}\right) / \partial x_{2}<0\right)$. Wei et al. ${ }^{14}$ explored the mean momentum balance based on experimental and DNS data and proposed a four layer structure for wallbounded flows where the wall-normal extent of the layers depend on the relative magnitude of $T$ compared to the viscous term. Therefore, there is great interest in exploring the structure of $T$ to understand the motions that contribute to the mean-momentum balance.

The wall-normal gradient of Reynolds shear stress in an incompressible flow can be written as follows: ${ }^{15}$

$$
T=u_{2} \omega_{3}-u_{3} \omega_{2}+\frac{1}{2} \frac{\partial}{\partial x_{1}}\left(u_{1}^{2}-u_{2}^{2}-u_{3}^{2}\right)
$$

where, $\omega_{2}, \omega_{3}$ are the fluctuating vorticity components along $x_{2}$ and $x_{3}$ respectively.

Klewicki ${ }^{16}$ documented the individual terms that appear in equation 3 and concluded that the wallnormal gradient of $-\overline{u_{1} u_{2}}$ is dominated by the velocity-vorticity correlation terms. The streamwise gradients of the mean-square fluctuations (the term $C$ ) were generally over three order of magnitude smaller than the velocity-vorticity correlations. Therefore, equation 3 can be approximated as,

$$
T \approx u_{2} \omega_{3}-u_{3} \omega_{2}=[u \times \omega]_{1}
$$

where $[u \times \omega]_{1}$ is the streamwise component of the Lamb vector.

Therefore, the gradient of Reynolds shear stress arises through the interactions between velocity and vorticity. These interactions produce motions that are capable of acting as either momentum sources/sinks. A few researchers have investigated the motions that contribute to the velocity-vorticity correlation. Rajagopal \& Antonia ${ }^{17}$ studied the structure of velocity field associated with the spanwise vorticity field and reported cross correlations of $u_{2} \omega_{3}$ and $u_{1} \omega_{3}$. They concluded that the velocity signature is consistent with the presence of internal shear layers, inclined to the wall. Klewicki et al. ${ }^{18}$ investigated the $u_{2} \omega_{3}$ term in the near wall region and concluded that the existence of positive spanwise vorticity for $x_{2}{ }^{+}>12$ is consistent with the presence of a ring-like eddy. ${ }^{19}$ Priyadharshana et al. ${ }^{20}$ compared the velocity-vorticity correlations over a wide range of Reynolds numbers and found that the interaction between velocity and vorticity depends on the Reynolds number and wall-roughness. They proposed a model where approximately uniform momentum zones (consistent with VLSMs) are intermittently segregated by narrow fissures of highly vortical flow and that the interaction between these vorticity fissure and velocity zones create momentum sources and sinks. However, the spatial distribution of these momentum source/sink like motions and their relationship with the Reynolds shear stress events is not documented.

In the current study, the spatial structure of momentum souces/sinks is explored by using dual-plane particle image velocimetry data that were obtain in streamwise-spanwise planes of a turbulent boundary layer. The primary goal is to determine the spatial structure of the streamwise component of the Lamb vector $\left([u \times \omega]_{1}\right)$ and classify the motions that act as momentum sources and sinks.

\section{Experimental facility and Details}

The experimental datasets analysed in this study were obtained by ${ }^{23}$ by performing dual-plane particle image velocimetry measurements in a suction-type boundary layer wind tunnel at the department of Aerospace Engineering and Mechanics, University of Minnesota. Measurements were performed in a zeropressure-gradient flow with freestream velocity $U_{\infty}=5.9 \mathrm{~m} \mathrm{~s}^{-1}$ and $R e_{\tau}=1200\left(R e_{\tau}=\delta U_{\tau} / \nu\right.$, where $\delta$ is the boundary layer thickness, $u_{\tau}$ is the skin friction velocity and $\nu$ is the kinematic viscosity). All quantities presented in this paper are non-dimensionalised using $u_{\tau}$ and $\nu$. The Reynolds number based on the momentum thickness $\operatorname{Re}_{\theta}$ was 2800 and the value of $\delta$ in the region of the measurement planes was 70 $\mathrm{mm}$. Measurements were obtained at $x_{2}{ }^{+}=110\left(x_{2} / \delta=0.1, \log\right.$ region $)$ and at $x_{2}{ }^{+}=575\left(x_{2} / \delta=0.5\right.$, outer wake region).

The experiments use a unique three-camera polarization-based dual-plane PIV system to measure the complete velocity gradient tensor. A schematic of the experimental setup is shown in figure 1 . The setup consists of two independent PIV systems that captures images of light scatter by olive oil droplets of size $1 \mu \mathrm{m}$. 


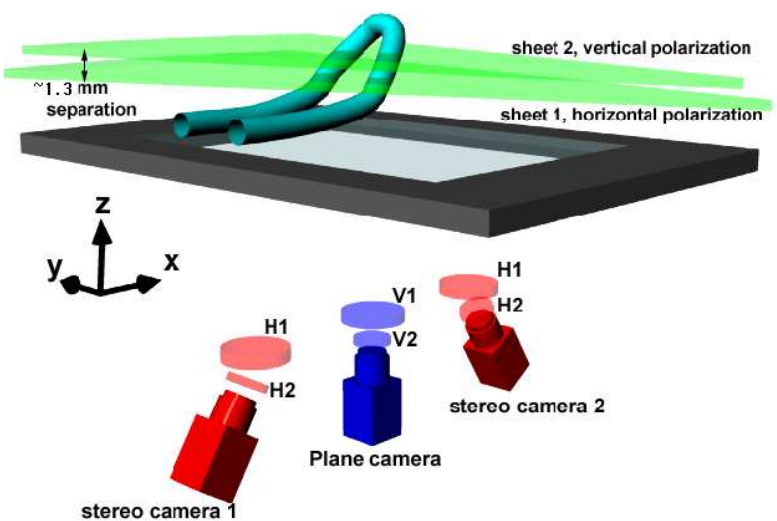

(a)
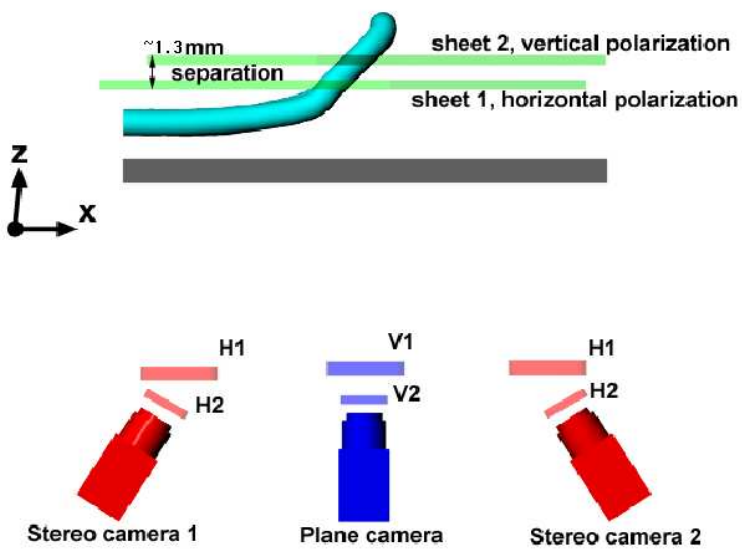

(b)

Figure 1. Schematic of the dual-plane PIV experimental setup. Figure reproduced from Ganapathisubramani et al. $^{21,22}$

The first system is a stereoscopic setup, which provided all three velocity components within a $x_{1}-x_{3}$ plane, while the second system was a conventional PIV system that measured the in-plane velocity components in a neighbouring $x_{1}-x_{3}$ plane located 20 wall units above in the wall-normal direction. Simultaneous measurements were performed in the two planes by using the polarization property of laser light sheets to isolate one camera system from the other. The images were analysed and velocity vectors were computed in both planes. The resolution of the resulting vector fields was about $12 \times 12$ wall units, and the total field size was $1.1 \delta \times 1.1 \delta$.

The instantaneous complete velocity gradient tensor was computed using the velocity data from both planes. A second order central difference method was used to compute all possible in-plane gradients while a first order forward difference was used to compute the wall-normal gradients. Further details of the experimental set up and validation of the velocity gradient and vorticity data are given in Ganapathisubramani et $a l^{21,22}$

\section{Results}

Quantitative analysis of the Lamb vector can be performed by computing probability density functions of $[u \times \omega]_{1}{ }^{+}$(superscript + indicates normalisation by $\nu$ and $u_{\tau}$ ). Figures 2(a) and 2(b) show the probability density functions $(p d f s)$ for $[u \times \omega]_{1}{ }^{+}$at $x_{2}{ }^{+}=110\left(x_{2} / \delta=0.1\right)$ and $575\left(x_{2} / \delta=0.5\right)$, respectively. The $p d f s$ are plotted in linear-log axes. Both figures indicate that the $p d f s$ are skewed towards negative values of $[u \times \omega]_{1}{ }^{+}$. The tails of the distribution exhibit exponential decay as evidenced by the straight lines fitted to the positive and negative tails in both figures. The constants that were used for the exponential fit are indicated in the figures. It is clear from the figures that the range of $[u \times \omega]_{1}{ }^{+}$values decrease with increasing wall-normal location. The slope of the exponential decay is found to be steeper away from the wall as evidenced by the value of the slope $k$. (where $k=15$ and 21 for $x_{2}{ }^{+}=110$ and 575 , respectively). The $p d f \mathrm{~s}$ deviate from the exponential decay for large negative values of $T^{+}$indicating a preference for large negative values of $[u \times \omega]_{1}{ }^{+}$at both wall-normal locations.

The location of the peak in the wall-normal profile of the Reynolds shear stress is found to be Reynolds number dependent. Sreenivasan \& Sahay ${ }^{24}$ presented a curve fit for the location of the peak based on the Karman number: $x_{2 p}{ }^{+} \approx 2 \sqrt{R e_{\tau}}$. Alternately, Fernholz \& Finley ${ }^{25}$ examined experimental data over a wide range of Reynolds numbers and found that the peak location varies as $x_{2 p}{ }^{+} \approx R_{\theta}{ }^{0.61}$. Substituting the relevant values in the functional form, the peak in the mean Reynolds shear stress profile for the boundary layer in the current study is expected to be located between 70 wall-units to 100 wall-units. This indicates that both measurement planes are likely to be located beyond the peak location. This is reflected in the mean values of $[u \times \omega]_{1}{ }^{+}$, which are -0.006 and -0.008 at $x_{2}{ }^{+}=110$ and 575 , respectively. These values are consistent with the values reported in previous studies in the literature. ${ }^{16,20}$ 

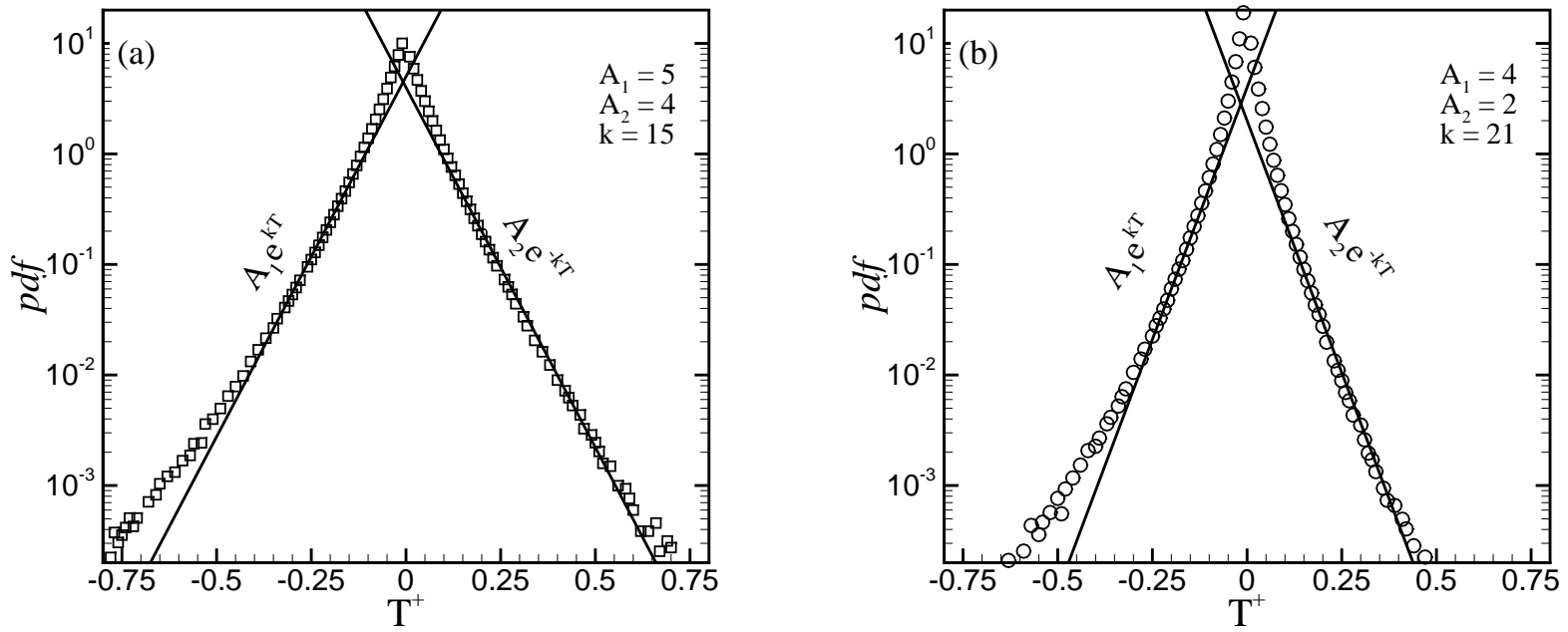

Figure 2. Probability density functions of $[u \times \omega]_{1}^{+}$at $(a) x_{2}{ }^{+}=110$ and $(b) x_{2}{ }^{+}=575$. The lines show the exponential decay of the values.

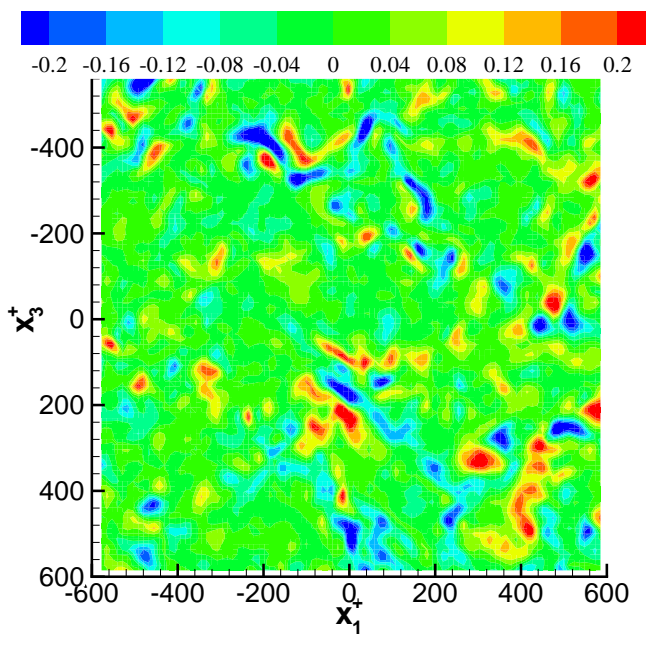

(a)

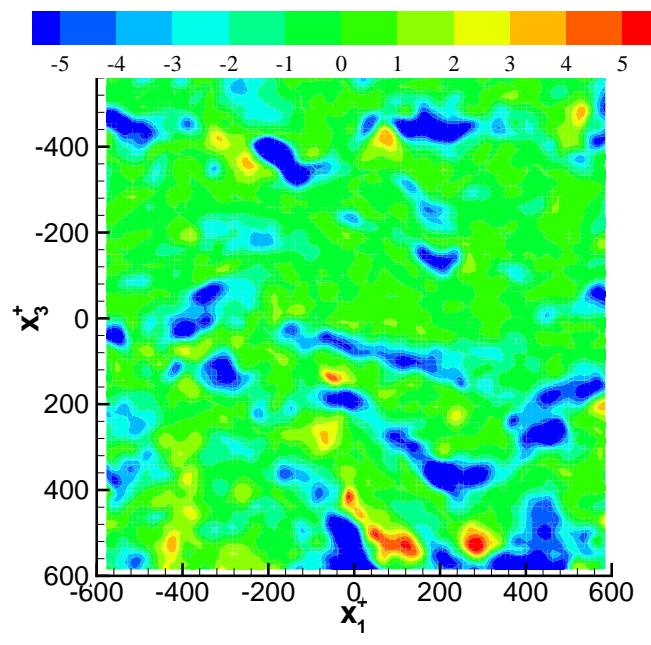

(b)

Figure 3. Instantaneous contours of (a) streamwise component of the Lamb vector $\left([u \times \omega]_{1}{ }^{+}\right)$, and (b) Reynolds shear stress $\left(\left[u_{1} u_{2}\right]^{+}\right)$. 
Figures 3(a) shows contours of the streamwise component of the Lamb vector $\left([u \times \omega]_{1}^{+}\right)$in a streamwisespanwise plane at $x_{2}{ }^{+}=110$. The contours indicate that $[u \times \omega]_{1}{ }^{+}$has large quiescent zones with intermittent areas of intense values. The intense regions possess both positive and negative values indicating the presence of both momentum source- and sink-like behaviour. These momentum sources and sinks appear to be located adjacent to each other. Figures 3(b) reveal instantaneous contours of Reynolds shear stress $\left(\left[u_{1} u_{2}\right]^{+}\right)$at the same instant as in figure 3(a). The figure shows that intense values of Reynolds shear stress is also intermittent. A comparison between figures 3(a) and 3(b) indicates that the momentum sources and sinks appear in areas where the Reynolds shear stress is negative. This indicates that the momentum sources/sinks are prevalent in ejection $\left(u_{1}<0\right.$ and $\left.u_{2}>0\right)$ and sweep $\left(u_{1}>0\right.$ and $\left.u_{2}<0\right)$ regions.

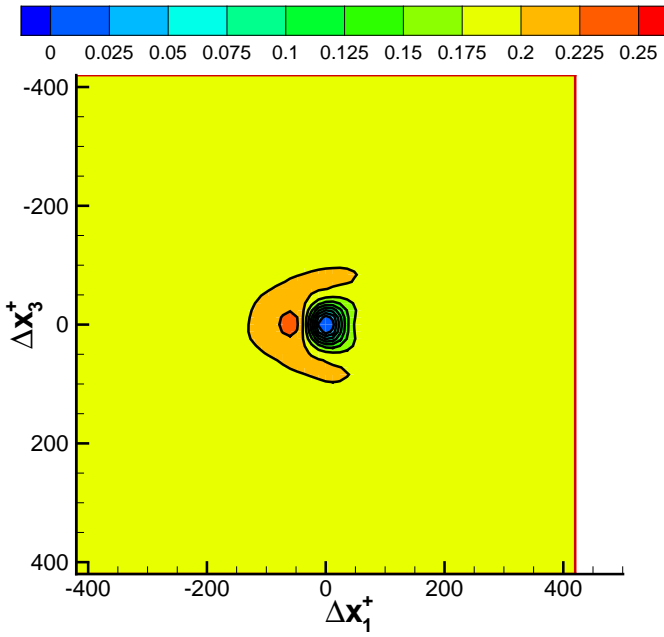

(a)

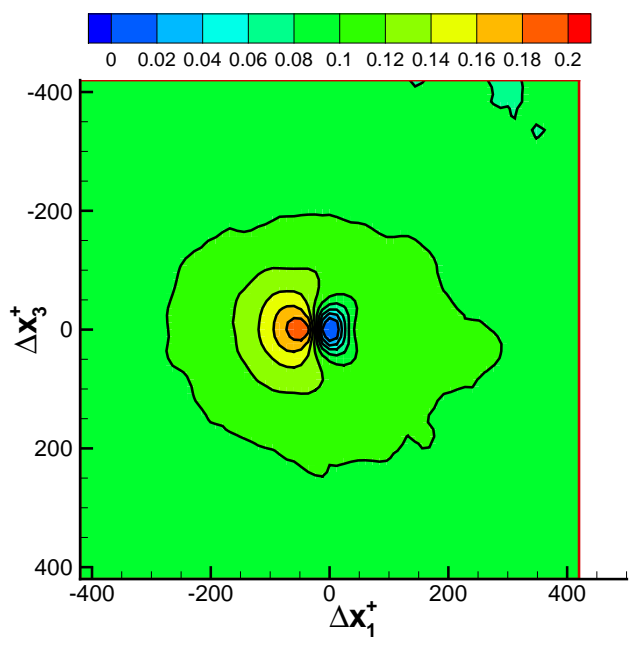

(b)

Figure 4. Probability of occurrence of momentum sinks in the vicinity of momentum sources $(\mathrm{a}) x_{2}{ }^{+}=110$ and (b) $x_{2}{ }^{+}=\mathbf{5 7 5}$.

The structure of $[u \times \omega]_{1}^{+}$can be further explored by computing the probability of occurrence of momentum source/sink events in the vicinity of each other. Figures 4(a) shows the probability distribution of locating a momentum sink in the neighbourhood of a momentum source at $x_{2}{ }^{+}=110$. The figure shows that the highest probability of locating a momentum sink is in a region that is approximately 60 wall-units downstream of a momentum source. Additionally, the probability is also high in a region that is 75 wall-units away in the either spanwise directions. However, the spanwise extent of these high probability regions are small. In fact, the figure reveals that the region of high probability possesses a 'boomerang' shape and momentum sources are encompassed by momentum sinks in all directions except in the upstream direction.

Figure 4(b) shows the probability distribution of locating a momentum sink in the neighbourhood of a momentum source at $x_{2}{ }^{+}=575$. This figure shows that the highest probability of locating a momentum sink in the vicinity of a momentum source is at a downstream distance of approximately 60 wall-units. This suggests that the spatial separation between momentum sources/sinks does not change with wall-normal location. However, the shape of the high probability region appears to be different in this outer location. The highest probability is confined to the downstream direction.

The relationship between the Lamb vector (i.e. Momentum sources/sinks) and Reynolds shear stress can be explored by computing the joint pdf between the two quantities. In fact, the covariance between these two quantities will shed insights in to their relationship. The covariance between any two variables is derived from the joint pdf using the following equation,

$$
C O V(A, B)=\int A B P(A, B) d A d B
$$

where, $P(A, B)$ is the joint pdf between the variables $A$ and $B$.

Figures 5(a) and 5(b) show the covariance maps between the Lamb vector and Reynolds shear stress at $x_{2}{ }^{+}=110$ and 575, respectively. Both figures show strong correlation between $[\vec{u} \times \vec{\omega}]_{1}<0$ and $u_{1} u_{2}<0$ 


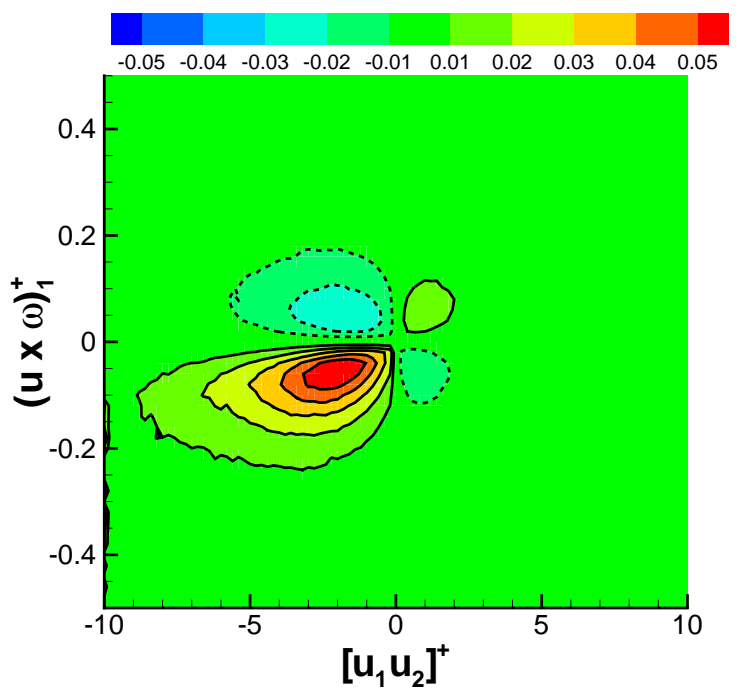

(a)

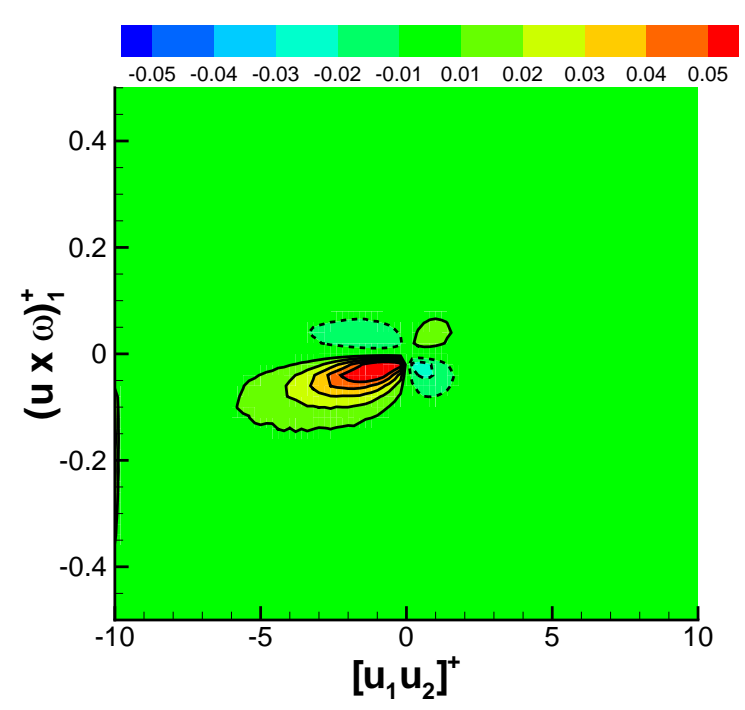

(b)

Figure 5. Covariance between the Lamb vector and Reynolds shear stress at (a) $x_{2}^{+}=\mathbf{1 1 0}$ and (b) $x_{2}{ }^{+}=\mathbf{5 7 5}$.

in both wall-normal locations. This suggests that momentum sources and sinks predominantly occur in regions of sweeps (Q4 events) and ejections (Q2 events). This is consistent with the observation based on instantaneous fields in figure 3. Although the covariance maps indicate the relationship between the two quantities, the characteristics of Reynolds shear stress in the vicinity of momentum source/sinks cannot be inferred from these plots. Therefore, an alternate diagnostic is required to derive this information.

Probability of occurrence distribution between the Lamb vector and Reynolds shear stress will be able to provide further insights in to the flow fields that act as momentum sources and sinks. These occurrence probabilities must be calculated for both momentum sources and sinks and must account for the four independent quadrants of Reynolds shear stress.

Figure 6(a)-6(d) shows the probability of occurrence of Q1, Q2, Q3 and Q4 events in the vicinity of momentum source at $x_{2}{ }^{+}=110$. Figure 6(a) indicates that the highest probability of locating Q1 events in the vicinity of momentum sources is in a pear-shaped region around the momentum source. Beyond this region, the probability of of locating a Q1 events appears to be homogeneous in all directions. Figure 6(b) indicates that Q2 events possess the highest probability of occurrence in the neighbourhood of momentum sources. The figure shows that this region extend in both upstream and downstream of the origin along the streamwise direction (to distances over 200 wall-units on either side). The figure also indicates that the spanwise width of this region is approximately 300 wall-units at this wall-normal location. The occurrence probability sharply decreases in the spanwise direction at a distance of approximately 200 wall-units. Figure 6(d) indicates that Q4 events possess the highest probability occurrence in the area where a sharp decrease in Q2 activity is observed. Finally, outward interactions (Q3 events) does not reveal any preference in its location as seen in figure 6(c). Similar distributions are observed in the outer wall-normal location $\left(x_{2}{ }^{+}=\right.$ 575 , not shown here).

Figure 7(a)-7(d) shows the probability of occurrence of Q1, Q2, Q3 and Q4 events in the vicinity of momentum sinks at $x_{2}{ }^{+}=110$. Figure 7 (a) shows that Q1 events do not have a preference to appear in the vicinity of momentum sinks. In fact, the probability decreases near the origin (i.e. at the same point as a momentum sink). This is consistent with the observations based on the covariance maps in figure 5(a). Ejections events reveal a strong preference to be located in the neighbourhood of momentum sink as seen in figure 7(b). The region of highest probability possesses a wedge shape that extends approximately 100 wall-units in the upstream and downstream directions. The spanwise width of this wedge-shaped region decreases along the streamwise direction from 400 wall-units at $\Delta x_{1}{ }^{+}=-100$ to 100 wall-units at $\Delta x_{1}{ }^{+}$ $=100$. Figure $7(\mathrm{c})$ reveals that the probability of finding a concurrent Q3 event-momentum sink is low. However, the Q3 events appear to be located upstream and downstream of momentum sinks. These regions of high probability are located at a distance of \pm 50 wall-units along the streamwise direction. The spanwise 


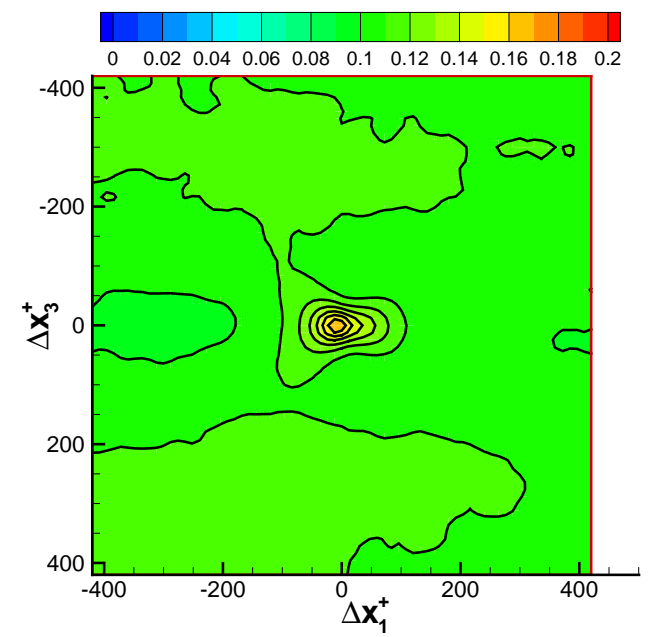

(a)

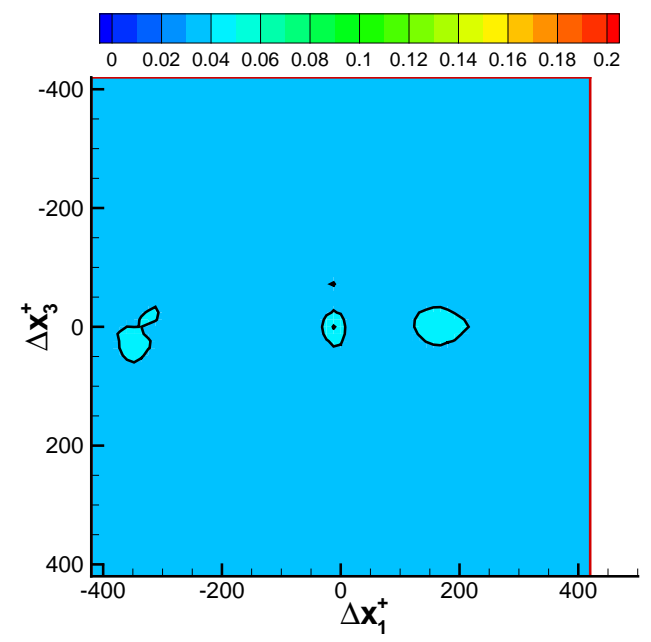

(c)

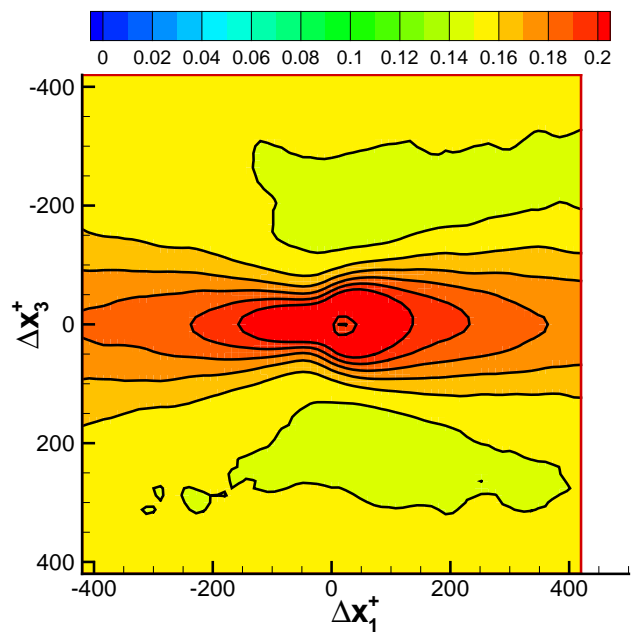

(b)

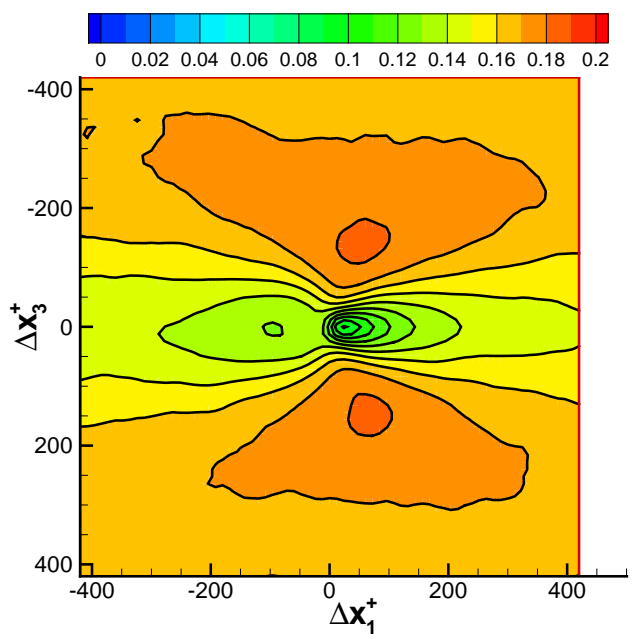

(d)

Figure 6. Probability of occurrence of Reynolds shear stress events in the vicinity of momentum sources at $x_{2}{ }^{+}$ = 110. (a) Q1 (inward interactions), (b) Q2 (ejections), (c) Q3 (outward interactions) and (d) Q4 (sweeps). 


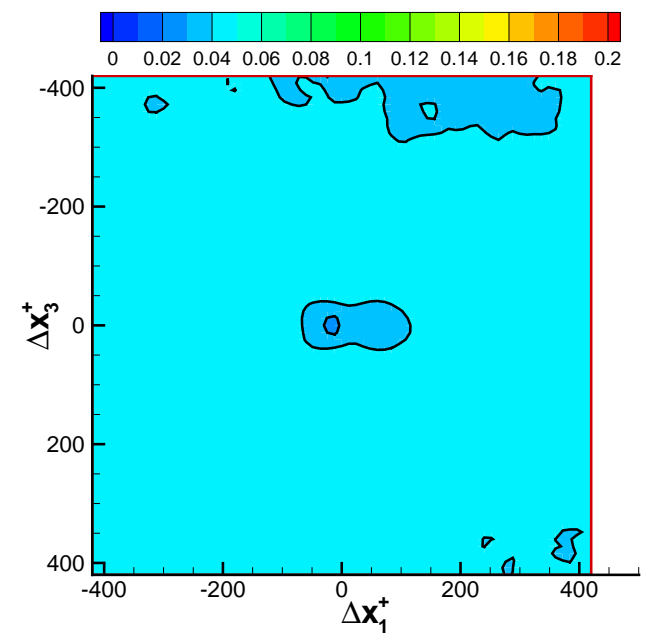

(a)

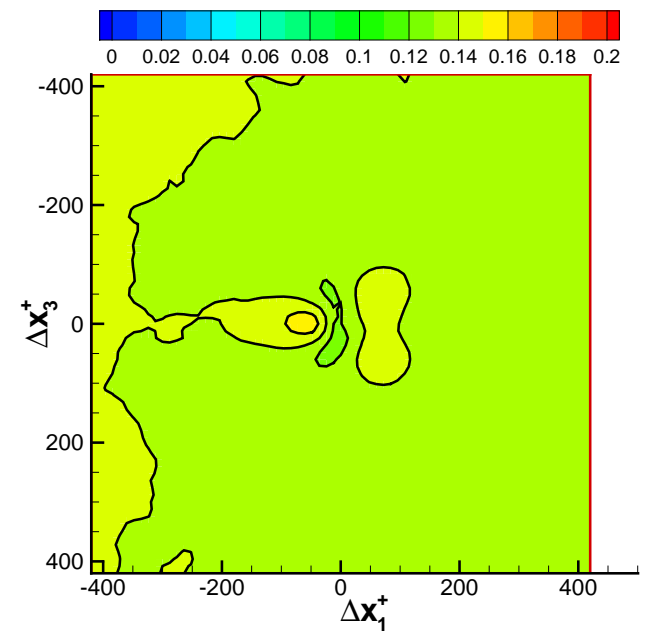

(c)

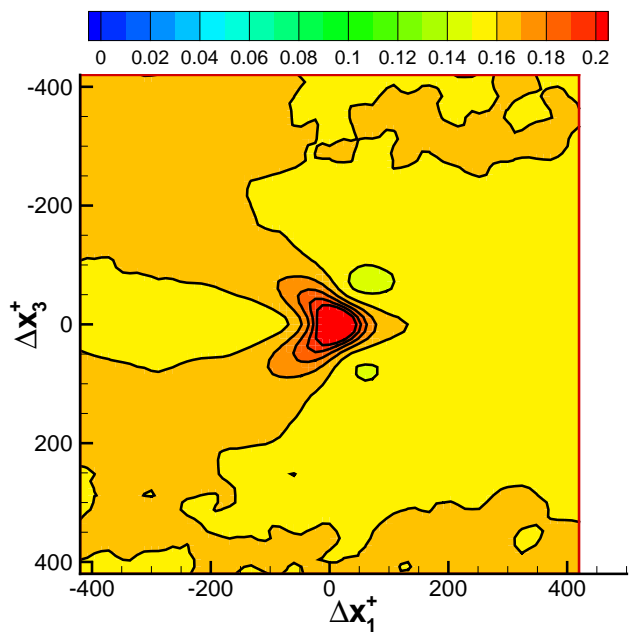

(b)

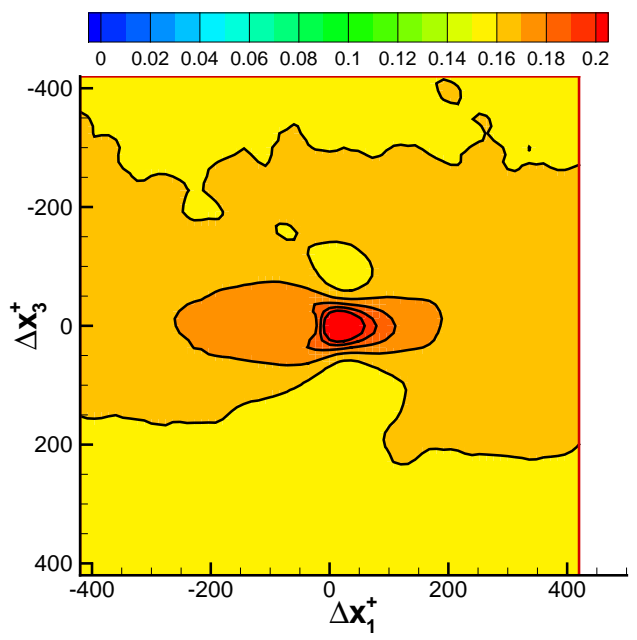

(d)

Figure 7. Probability of occurrence of Reynolds shear stress events in the vicinity of momentum sources at $x_{2}{ }^{+}$ = 110. (a) Q1 (inward interactions), (b) Q2 (ejections), (c) Q3 (outward interactions) and (d) Q4 (sweeps). 
width of these regions is approximately 100 wall-units. Finally, figure 7(d) shows that sweep events possesses highest probability in a region that extends along the streamwise direction.

\section{Summary and Conclusions}

The spatial organisation of the Lamb vector is examined in the outer region of a turbulent boundary layer. The streamwise component of the Lamb vector acts as a momentum source in areas where its value is greater than zero and as a momentum sink in regions where its value is less than zero. Inspection of instantaneous fields indicates that momentum-sources and sinks reveal an intermittent character and they appear adjacent to each other. Conditional probability analyses indicates that momentum source-like events predominantly occurs upstream of momentum sink-like events in both wall-normal locations examined in this study.

The spatial layout and the relationship between momentum sources/sinks and Reynolds shear stress is explored by computing covariance maps and two-dimensional probability of occurrence distribution. The results indicate that momentum sources/sinks appear to in areas of negative Reynolds stresses. This suggests that turbulence production sites (i.e. areas of $u_{1} u_{2}<0$ ) contains both momentum sources and sinks. Within these regions, momentum sources largely lie within ejection-type events (Q2 events), while sweep events occur adjacent to momentum sources. Both inward and outward interactions do not display any directional or location preference in the vicinity of momentum sources. Momentum sinks on the other hand, reveals comparable association with both sweep and ejection events. The region in the vicinity of momentum sinks also appears to be sites of outward interactions (Q3 events). This suggests that momentum sinks appears in areas where rapid quadrant transitions take place and this is consistent with previous studies that has explored the nature of momentum sources and sinks. ${ }^{26}$

\section{Acknowledgements}

The author is grateful to Professor E. K. Longmire and Professor I. Marusic for all their support and Professor J. Klewicki for thought provoking discussions.

\section{References}

${ }^{1}$ Willmarth, W. W. and Lu, S. S., "Structure of the Reynolds stress near the wall," J. Fluid Mech., Vol. 55, 1972, pp. 65-92.

${ }^{2} \mathrm{Lu}$, S. S. and Willmarth, W. W., "Measurements of the structure of the Reynolds stress in a turbulent boundary layer," J. Fluid Mech., Vol. 60, 1973, pp. 481-511.

${ }^{3}$ Blackwelder, R. F. and Kaplan, R. E., "On the wall structure of the turbulent boundary layer," J. Fluid Mech., Vol. 76-1, 1976, pp. 89-120.

${ }^{4}$ Wallace, J. M. and Brodkey, R. S., "Reynolds stress and joint probability density distributions of the $u-v$ plane of a turbulent channel flow," Phys. Fluids, Vol. 20, 1977, pp. 351-355.

${ }^{5}$ Theodorsen, T., "Mechanism of turbulence," Proc. Second Midwestern Conference on Fluid Mechanics, Mar. 17-19, Ohio state Univesity, Columbus, Ohio, 1952.

${ }^{6}$ Offen, G. R. and Kline, S. J., "A proposed model of the bursting process in turbulent boundary layer," J. Fluid Mech., Vol. 70-2, 1975, pp. 209-228.

${ }^{7}$ Bandyopadhyay, P., "Large structure with a characteristic upstream interface in turbulent boundary layers," Phys. Fluids, Vol. 23-11, 1980, pp. 2326-2327.

${ }^{8}$ Head, M. R. and Bandyopadhyay, P., "New aspects of turbulent boundary-layer structure," J. Fluid Mech., Vol. 107, 1981, pp. 297-337.

${ }^{9}$ Adrian, R. J., Meinhart, C. D., and Tomkins, C. D., "Vortex organization in the outer region of the turbulent boundary layer," J. Fluid Mech., Vol. 422, 2000, pp. 1-53.

${ }^{10} \mathrm{Na}$, Y., Hanratty, T. J., and Liu, Z., "The use of DNS to define stress producing events for a turbulent channel flow over a smooth wall," Flow Turbul. Combust., Vol. 66, 2001, pp. 495-512.

${ }^{11}$ Ganapathisubramani, B., Longmire, E. K., and Marusic, I., "Characteristics of vortex packets in turbulent boundary layers," J. Fluid Mech., Vol. 478, 2003, pp. 35-46.

${ }^{12}$ Guala, M., Hommema, S. E., and Adrian, R. J., "Large-scale and very-large-scale motions in turbulent pipe flow," J. Fluid Mech., Vol. 554, 2006, pp. 521-542.

${ }^{13}$ Adrian, R. J., "Hairpin vortex organization in wall turbulence," Phys. Fluids, Vol. 19, 2007, pp. 041301.

${ }^{14}$ Wei, T., Fife, P., Klewicki, J. C., and McMurtry, P., "Properties of mean momentum balance in turbulent boundary layer, channel and pipe flows," J. Fluid Mech., Vol. 522, 2005, pp. 303-327.

${ }^{15}$ Hinze, J. O., Turbulence, McGraw-Hill, New York., 1975. 
${ }^{16}$ Klewicki, J. C., "Velocity-Vorticity correlations related to the gradients of the Reynolds stresses in parallel turbulent wall flows," Phys. Fluids, Vol. 1(7), 1989, pp. 1285-1288.

${ }^{17}$ Rajagopalan, S. and Antonia, R. A., "Structure of the velocity field associated with the spanwise vorticity in the wall region of a turbulent boundary layer," Phys. Fluids, Vol. 5(10), 1993, pp. 2502-2510.

${ }^{18}$ Klewicki, J. C., Murray, J. A., and Falco, R. E., "Vortical motion contributions to stress transport in turbulent boundary layers," Phys. Fluids, Vol. 6(1), 1994, pp. 277-286.

${ }^{19}$ Falco, R. E., "A Coherent Structure Model of the Turbulent Boundary Layer and Its Ability to Predict Reynolds Number Dependence," Phil. Trans. R. Soc. A, Vol. 336, 1991, pp. 103-129.

${ }^{20}$ Priyadarshana, P. J. A., Klewicki, J. C., Treat, S., and Foss, J., "Statistical structure of turbulent boundary layer velocity-vorticity products at high and low Reynolds numbers," J. Fluid Mech., Vol. 570, 2007, pp. 307-346.

${ }^{21}$ Ganapathisubramani, B., Longmire, E. K., Marusic, I., and Pothos, S., "Dual-plane PIV technique to measure complete velocity gradient tensor in a turbulent boundary layer," Exp. Fluids, Vol. 39(2), 2005, pp. 222-231.

${ }^{22}$ Ganapathisubramani, B., Longmire, E. K., and Marusic, I., "Experimental investigation of vortex properties in a turbulent boundary layer," Phys. Fluids, Vol. 18, 2006, pp. 055105.

${ }^{23}$ Ganapathisubramani, B., Investigation of turbulent boundary layer structure using stereoscopic particle image velocimetry, Ph.D. thesis, University of Minnesota, USA, 2004.

${ }^{24}$ Sreenivasan, K. R. and Sahay, A., "The persistence of viscous effects in the overlap region, and the mean velocity in turbulent pipe and channel flows," Self-Sustaining Mechanisms of Wall Turbulence, Ed. R.L. Panton, Comp. Mech. Publications, 1997.

${ }^{25}$ Fernholz, H. H. and Finley, P. J., "The incompressible zero-pressure-gradient turbulent boundary layer: An assessment of the data," Prog. Aerosp. Sci., Vol. 32, 1996, pp. 25-311.

${ }^{26}$ Ganapathisubramani, B., "Statistical structure of momentum sources and sinks in the outer region of a turbulent boundary layer," J. Fluid Mech., Vol. 606, 2008, pp. 225-237. 